\title{
Ultrasonic Guided Wave Method For Crack Detection In Buried Plastic Pipe
}

\author{
Wan Sofian Wan Hamat ${ }^{1, a}$, Mohd Fairusham Ghazali ${ }^{1}$ and Gigih Priyandoko ${ }^{1}$ \\ ${ }^{1}$ Faculty of Mechanical Engineering, Universiti Malaysia Pahang, 26600 Pekan, Pahang, Malaysia
}

\begin{abstract}
Plastic pipe are widely used in many fields for the fluid or gaseous product conveyance but basic components of a plastic material made it very sensitive to damage, which requires techniques for detecting damage reliable and efficient. Ultrasonic guided wave is a sensitive method based on propagation of low-frequency excitation in solid structures for damage detection. Ultrasonic guided wave method are performed to investigate the effect of crack to the frequency signal using Fast Fourier Transform (FFT) analysis. This paper researched to determine performance of ultrasonic guided wave method in order to detect crack in buried pipeline. It was found that for an uncrack pipe, FFT analysis shows one peak which is the operating frequency by the piezoelectric actuator itself while the FFT analysis for single cracked pipe shows two peak which is the operating frequency by the piezoelectric actuator itself and the resultant frequency from the crack. For multi cracked pipe, the frequency signal shows more than two peak depend the number of crack. The results presented here may facilitate improvements in the accuracy and precision of pipeline crack detection.
\end{abstract}

\section{Introduction}

Recent developments in pipelines systems have heightened the need for study about the pipe defect detection using ultrasonic guided wave method. In recent years, several studies have focused on defect detection in pipeline systems because it is very important used extensively all over the world to transport and distribute natural gas, crude oil, water, and other easy-flowing products. Indeed, in the process of continuous transportation, gas pipeline leakage cannot be avoided because of the corrosion of pipe walls [1], third-party interference [2] , aging of the pipes [3] , and so on. Therefore, leakage detection and localization is one of the paramount concerns of pipeline operators and researchers all over the world.

Many researchers have argued that Non-destructive testing (NDT) is a wide group of analysis techniques used in science and industry to evaluate the properties of a material, component or system without causing damage. NDT is a highly valuable technique that can save both money and time in product evaluation, troubleshooting, and research. Common NDT methods include ultrasonic, magnetic-particle, liquid penetrant, radiographic, remote visual inspection (RVI), eddy-current testing, and low coherence interferometry. According to K Haller (2007), damage can be monitored in many ways [4].
The choice of composite materials is no longer restricted to specific applications. In other fields, using these new materials as innovation tool and performance rising of their products such civil engineering. It is important first to have the best possible knowledge about these materials in somehow to monitor any changes in these properties and consequently their influence on the structure behavior $[\underline{5}, \underline{6}]$.

Plastic pipe is a good material because high resistance, light, cheap and longer life span. Therefore, plastic pipes usually are used to transport gas, water and chemicals. In general, plastic pipes are widely used over metal or concrete pipes. Due to the material being immune to water corrosion and highly resistant to fouling, it is also being considered as a replacement for stainless steel in safety-critical applications in nuclear power stations [7].

According to Carvalho et al. (2008), researchers have spent an ample amount of time, investigations and researched on non-destructive test techniques. As much as during the setting up phase during their useful life, these techniques are used as a method to asses engineering structures and system [] $]$. Crack inspection is a valuable non-destructive test (NDT) method in many industries. Crack inspection method is useful, which often exhibit complex geometries, are exposed to high thermal and mechanical loads and are expected to satisfy

\footnotetext{
${ }^{a}$ Corresponding author: w_sofian45@yahoo.com
} 
strict safety requirements. Lately, there is a need to expand a powerful crack defect detection methods [9]

Early detection of damage is not necessarily visible from the external face and even when it is the case of visual inspection does not realize its case state. In order of reliable and efficient use, these new materials, the development of a measurement system that can answer the following two questions of primary importance is required whether it could be measured directly in NDT and reliable mechanical properties of a composite structure. Is it possible to detect damage as quick as possible in order to monitor, evaluate and repair if necessary the structure [10]. Piezoelectric materials constitute another important research topic with respect to Structural Health Monitoring (SHM) applications. Piezoelectric transducers are often used to excite and receive ultrasonic guided waves due to their low costs and an easy integration into existing structures. This research area offers a wide variety of contributions [11, 12].

Although there were many researches about ultrasonic guided wave method to detect pipe damage, few of them focused on result obtained only for this method. So it is necessary to do deep research on the design and develop an experimental test rig for pipe defect detection to run easily, faster, efficient and cost-effective.

\section{Experimental Set Up}

\section{Design of Experiment}

The aim of this paper is to determine the performance of ultrasonic guided wave method in detecting cracks in the pipeline system using an experiment. An experimental test rig design and develop for buried plastic pipe defect detection to prevent the leakage in the piping system. All the experiments were conducted on plastic pipe specimens with piezoelectric wafer actuator (PSI-5A4E) and accelerometer mounted on the surface of each pipe. Piezoelectric wafer actuator as a transmitter and the accelerometer as a receiver. A National Instruments Data Acquisition system (NI USB-4431) was used to drive the transmitter and to record data and waveform at the receiver. The specifications of the experimental specimens are given in Table 1.
Table 1. Specification of the experimental specimens

\begin{tabular}{|c|c|c|c|c|c|}
\hline \multirow{3}{*}{$\begin{array}{l}\text { Exp. } \\
\text { No. }\end{array}$} & \multicolumn{4}{|c|}{ Buried Plastic Pipe Specimens } & \multirow{3}{*}{$\begin{array}{c}\begin{array}{c}\text { Sensors } \\
\text { \& } \\
\text { Trans- } \\
\text { ducers }\end{array} \\
\begin{array}{l}\text { Dist. }^{c} \\
\text { [mm] }\end{array}\end{array}$} \\
\hline & \multicolumn{3}{|c|}{ Dimensions [mm] } & \multirow{2}{*}{$\begin{array}{l}\text { Specificati- } \\
\quad \text { ons }\end{array}$} & \\
\hline & Length & $\mathrm{OD}^{\mathrm{a}}$ & $\mathrm{WT}^{\mathrm{b}}$ & & \\
\hline 1.1 & 600 & 40 & 2 & \multirow{5}{*}{$\begin{array}{l}\text { Difference } \\
\text { distance } \\
\text { between } \\
\text { the } \\
\text { transmitter } \\
\text { and the } \\
\text { receiver. }\end{array}$} & 300 \\
\hline 1.2 & 600 & 40 & 2 & & 500 \\
\hline 1.3 & 1300 & 40 & 2 & & 600 \\
\hline 1.4 & 1300 & 40 & 2 & & 900 \\
\hline 1.5 & 1300 & 40 & 2 & & 1200 \\
\hline 2.0 & 600 & 40 & 2 & $\begin{array}{l}1 \text { lateral } \\
\text { saw cut. }\end{array}$ & 500 \\
\hline 3.0 & 600 & 40 & 2 & $\begin{array}{l}2 \text { lateral } \\
\text { saw cut. }\end{array}$ & 500 \\
\hline
\end{tabular}

$\left({ }^{\mathrm{a} O D}\right.$ : outside diameter; ${ }^{\mathrm{b}} \mathrm{WT}$ : wall thickness; ${ }^{\mathrm{c}} \mathrm{Dist}$ : distance between the transmitter and the receiver).

\section{Experimental Procedure}

This experiment carried out a few times to make sure a more accurate result is obtained. First, the experiment will be carried out on an uncrack specimen. Data will be collected and analyzed. Next, the experiment will be carried out on a specimen with a single crack. Lastly, the experiment will be carried out on a specimen with multi cracks. All the data were collected and analyzed. From the data collected, we can compare the result of all the three specimen.

Piezoelectric wafer actuator (PSI-5A4E) was used to excite the specimen. By placing the specimen on the foams and using a significantly smaller size of wafer actuator, this is the best compromise method for diagnosing defects. Nonlinear effects and noise effects from the attachment of the mechanical shaker or rigid supports can be prevented by using this method. A comparison was made between this method and the former method that using a mechanical shaker for the excitation. Although the maximum excitation level from the piezoelectric actuator is relatively lower than with the mechanical shaker, results from the latter method also showed a good signature of the sidebands effect against the defects $[\underline{10}, \underline{13}]$.

The experiment for uncrack specimen begin with distance between piezoelectric wafer actuator as a transmitter and accelerometer as a receiver is $300 \mathrm{~mm}$. The frequencies applied to the specimen are $500 \mathrm{~Hz}, 3000 \mathrm{~Hz}, 6000 \mathrm{~Hz}$, and $9000 \mathrm{~Hz}$. After the data is collected and recorded, the experiment is repeated with different value of distance 
between transmitter and receiver. This process is repeated with $600 \mathrm{~mm}, 900 \mathrm{~mm}$ and $1200 \mathrm{~mm}$ distance between transmitter and receiver. The relationship between the distance and amplitude for frequency signal can be identify from results obtained for this experiments.

The experiment for single crack specimen and multi cracks specimen was performed on a pipe with length $600 \mathrm{~mm}$ which the piezoelectric wafer actuator and accelerometer are placed on the surface of the pipe where the distance between each other is $500 \mathrm{~mm}$. Figure 1 illustrates the specimen for (a) uncrack specimen, (b) single crack specimen and (c) multi cracks specimen. Lateral saw cut made as a crack shown at Figure 1(b) and Figure 1(c). The crack made at the middle of this distance $(250 \mathrm{~mm})$ for single crack specimen; see Figure 1(b). For multi cracks specimen, two crack made at distance $165 \mathrm{~mm}$ from piezoelectric stack actuator and $165 \mathrm{~mm}$ from accelerometer; see Figure 1(c).

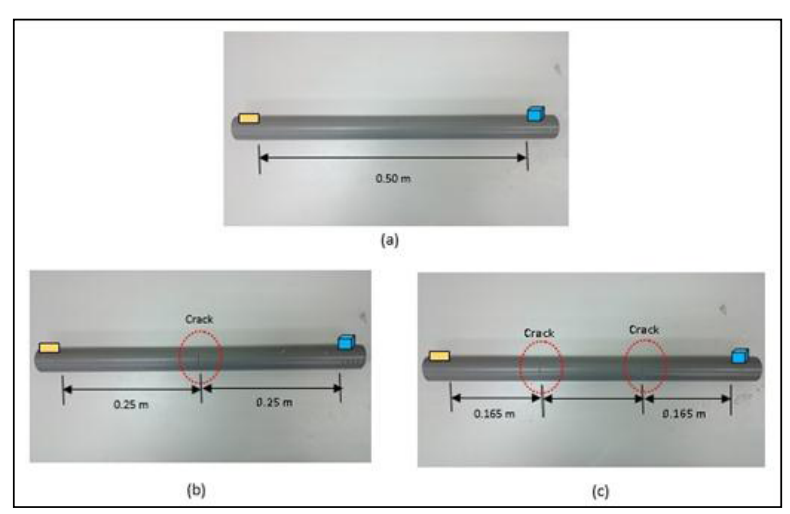

Figure 1. Piezoelectric wafer actuator and accelerometer placed on the surface of three pipe specimens. (a) Uncrack specimen; (b) Single crack specimen; (c) Multi cracks specimen.

The data collected will undergo analysis using the Dasylab and Matlab software. The data will also undergo amplification and filtering module. The amplification module is to amplify the data collected and filtering of the data is extremely vital in obtaining an accurate time base response graph. The plastic pipe will be attached the sensors and then it will be connected to Data Acquisition (DAQ) system. The Data Acquisition system will be synchronized to the Dasylab software using the NI-MAX software. The system will be configured to analyze the voltage as the sensor medium. The accelerometer will be controlled using the Dasylab software. After collecting the raw data, analyzing data were done by using Matlab software. The experiments setup for this study are illustrated in Figure 2(a) and Figure 2(b).

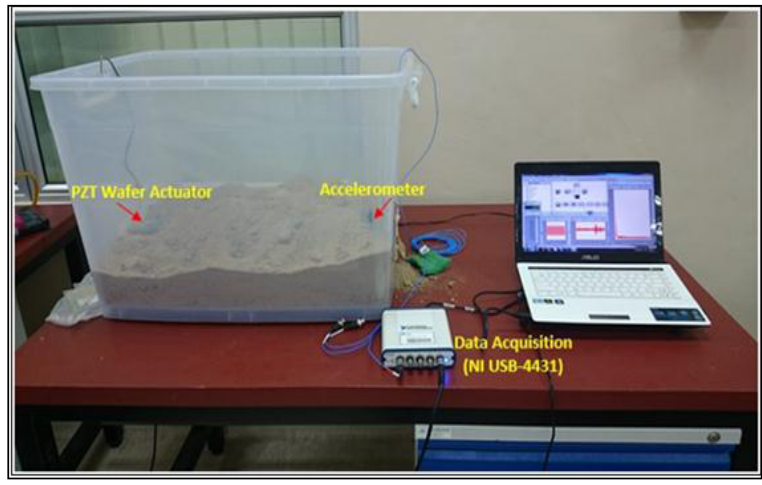

Figure 2(a). Full view for the experimental setup

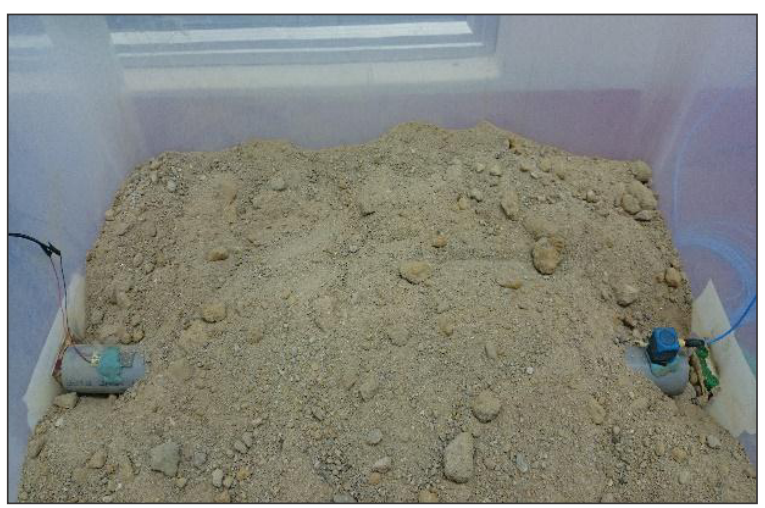

Figure 2(b). Buried pipe specimen

\section{Results and Discussion}

\section{The relationship between distance and amplitude}

The experiment results will be shown in the form of graph for Fast Fourier Transform (FFT) analysis which is produced by using Matlab software. Table 2 shows the comparison of amplitude value for each of the spectrum at different distance between sensor and actuator for uncrack specimen. The value of the amplitude decreases as the distance between actuator and sensor increases. This can be deduced that the waves tend to dissipate more when the distance traveled by the wave is increased. So, the waves will become weaker when the distance is increased and this explained the reason for the value of amplitude decreases when the distance increases. The amplitude value is inversely proportional with distance between sensor and actuator. 
Table 2. Comparison of amplitude value for each spectrum at different distance and frequency applied

\begin{tabular}{|c|c|c|c|c|}
\hline \multirow{2}{*}{$\begin{array}{c}\text { Frequency } \\
\text { (Hz) }\end{array}$} & \multicolumn{4}{|c|}{ Amplitude } \\
\cline { 2 - 5 } & $\begin{array}{c}\mathbf{3 0 0} \\
\mathbf{m m}\end{array}$ & $\begin{array}{c}\mathbf{6 0 0} \\
\mathbf{m m}\end{array}$ & $\begin{array}{c}\mathbf{9 0 0} \\
\mathbf{m m}\end{array}$ & $\begin{array}{c}\mathbf{1 2 0 0} \\
\mathbf{m m}\end{array}$ \\
\hline 3000 & 0.13267 & 0.06291 & 0.05385 & 0.02730 \\
\hline 6000 & 0.09017 & 0.04210 & 0.02834 & 0.01482 \\
\hline 9000 & 0.08762 & 0.00753 & 0.00376 & 0.00135 \\
\hline
\end{tabular}
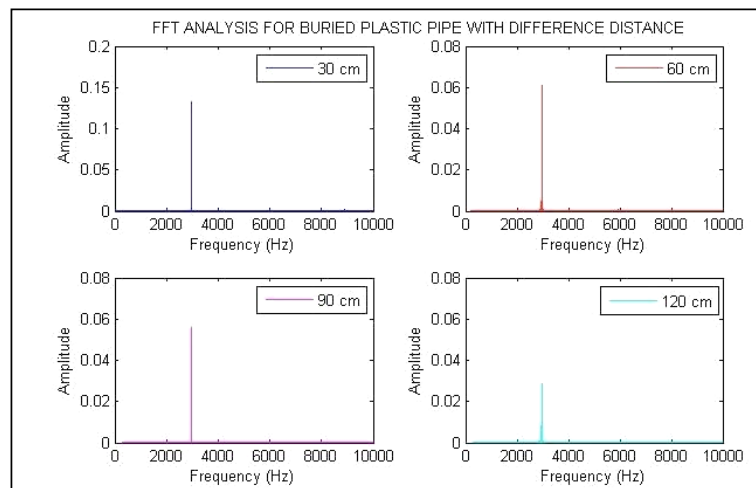

Figure 3. Results obtained for $3000 \mathrm{~Hz}$ frequency applied to the $300 \mathrm{~mm}, 600 \mathrm{~mm}, 900 \mathrm{~mm} 1200 \mathrm{~mm}$ of uncrack specimen.

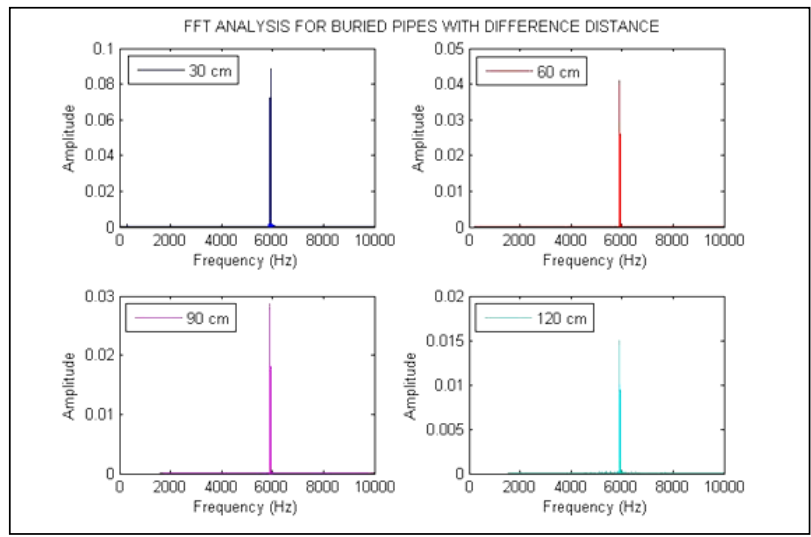

Figure 4. Results obtained for $6000 \mathrm{~Hz}$ frequency applied to the $300 \mathrm{~mm}, 600 \mathrm{~mm}, 900 \mathrm{~mm} 1200 \mathrm{~mm}$ of uncrack specimen.

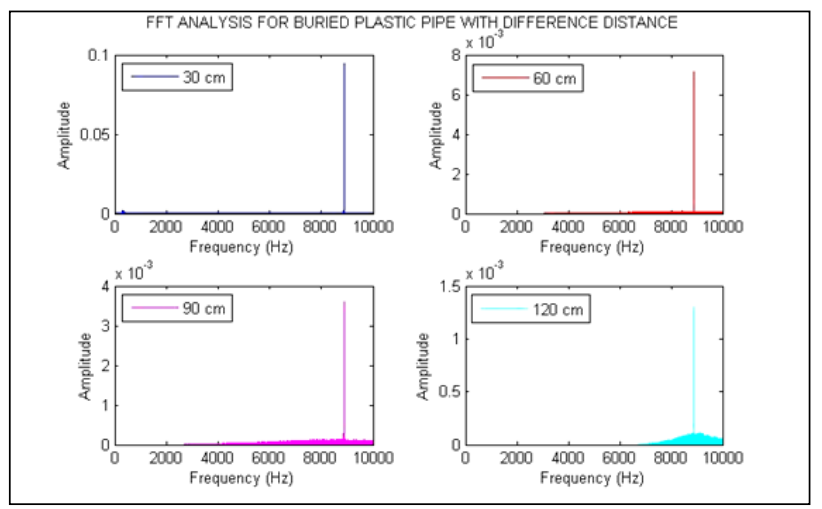

Figure 5. Results obtained for $9000 \mathrm{~Hz}$ frequency applied to the $300 \mathrm{~mm}, 600 \mathrm{~mm}, 900 \mathrm{~mm} 1200 \mathrm{~mm}$ of uncrack specimen.
From the FFT analysis graph in Figure 3, Figure 4, and Figure 5, it could be clearly seen that the amplitude of the frequency response signal decreases as the distance between the piezoelectric wafer actuator and accelerometer increases. For $3000 \mathrm{~Hz}$, the frequency signal response amplitude is 0.13267 at $300 \mathrm{~mm}$ followed by 0.06291 at $600 \mathrm{~mm}, 0.05385$ at $900 \mathrm{~mm}$ and 0.02730 at $1200 \mathrm{~mm}$; see Figure 3. For $6000 \mathrm{~Hz}$, the frequency signal response amplitude is 0.09017 at $300 \mathrm{~mm}$ followed by 0.04210 at $600 \mathrm{~mm}, 0.02834$ at $900 \mathrm{~mm}$ and 0.01482 at $1200 \mathrm{~mm}$; see Figure 4. For $9000 \mathrm{~Hz}$, the frequency signal response amplitude is 0.08762 at $300 \mathrm{~mm}$ followed by 0.00753 at $600 \mathrm{~mm}, 0.00376$ at $900 \mathrm{~mm}$ and 0.00135 at $1200 \mathrm{~mm}$; see Figure 5. All the frequencies show that that the frequency signal amplitude are the highest at the shortest distance. This shows that it is capable of travelling at a short distance.

In general, the frequency signal amplitude decreases in all samples due to the attenuation inside the testing medium as the distance from the source is increased too. It is clear that the attenuation increases with increasing distance. The transmitted signal is attenuated by the transmission medium which is the time required by the signal to reach the sensor point. This transmission makes some turbulence in the transmission medium and such turbulence may remain exist over this period of time. When transmission frequency increases, the transmission medium will contain effects of consecutive signals and constructive and destructive interferences would take place. Accordingly, the accelerometer would receive transmitted signals at a higher frequency and if the accelerometer needs longer time to distinguish the received signals from each other, then higher frequency may cause destructive interference at the sensor, which in turn affects the final recorded signal amplitude.

\section{Comparing the results for uncrack and crack specimen.}

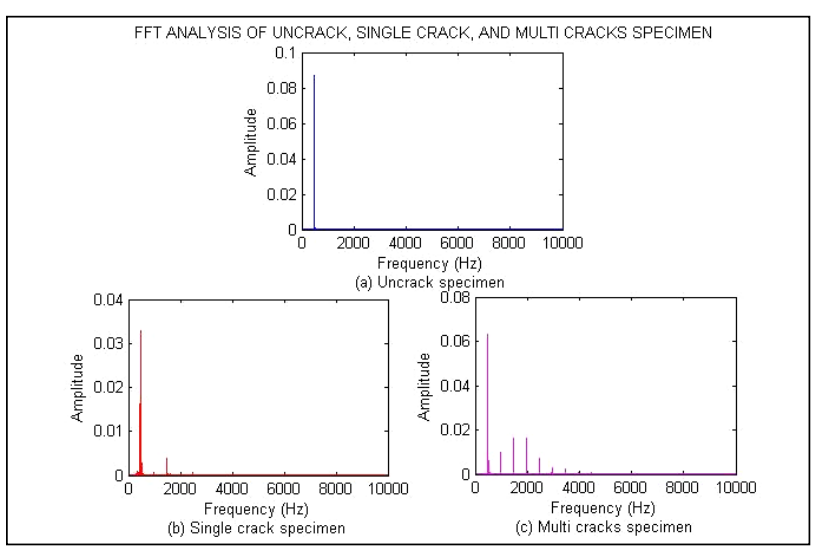

Figure 6. FFT analysis graph for (a) uncrack specimen, (b) single crack specimen, (c) multi cracks specimen. 
Figure 6(a) shows the FFT analysis graph of the results obtained for an uncrack specimen. From the results obtained, the FFT analysis graph shows one peak which is the operating frequency by the piezoelectric actuator itself. Figure 6(b) shows the FFT analysis graph for single crack specimen. The graph FFT analysis shows two peak which is the operating frequency by the piezoelectric wafer actuator itself and the resultant frequency from the crack. Figure 6(c) shows FFT analysis graph for multi cracks specimen. From the frequency signal obtained, the data shows more than two peaks which one peak is the operating frequency by the piezoelectric actuator itself and other peaks is the resultant frequency from the cracks.

Accelerometer has its own area of influence characterized by a sensing radius and the corresponding circle. Damage within the area of influence of the sensor is highly sensitive as it creates higher disturbances than the damage outside the area of influence. An exciting vibration at creates appreciable stress in the material affecting interfaces at all scales of damage. An ultrasound probe wave traveling through these interfaces is modulated due to vibration-induced changes in the interface condition and, as a result, additional spectral components appear at the frequencies. These new frequency components indicate that a flaw or crack is present.

\section{Conclusions}

This study set out to determine the performance of ultrasonic guided wave method in detecting cracks in the pipeline system using an experiment. An experimental test rig design and develop for buried plastic pipe defect detection to prevent the leakage in the piping system. The findings of this study suggest that detecting the defects in buried pipe using ultrasonic guided wave method is faster, efficient and cost-effective. From the data collected and analyzed, uncrack and crack specimen can be distinguished more obviously in a short time. The FFT analysis graph clearly shows only one peak for the uncrack specimen and two peak for the single crack specimen. There are more than two peak for the multi cracks specimen. Further investigation and experimentation for defect detection in pipe is strongly recommended. A number of possible future studies using the same experimental set up are apparent. It would be interesting to assess the effects of ultrasonic guided wave detecting the defect in pipe.

\section{Acknowledgements}

The work presented in this paper was supported by Universiti Malaysia Pahang (UMP) through the RDU Grant research project (RDU130111) lead by Dr. Mohd Fairusham Bin Ghazali. The authors are grateful to the Universiti Malaysia Pahang and Education Sponsorship
Division, Ministry Of Education Malaysia for supporting the present study.

\section{References}

1. Edalati K, R.N., Kermani A, Seidi M, Movafeghi A, The use of radiography for thickness measurement and corrosion monitoring in pipes. International Journal of Pressure Vessels and Piping, 2006. 83: p. 736-741.

2. Liang, W., et al., Assessing and classifying risk of pipeline third-party interference based on fault tree and SOM. Engineering Applications of Artificial Intelligence, 2012. 25(3): p. 594-608.

3. Vargas-Arista, B., J.M. Hallen, and A. Albiter, Effect of artificial aging on the microstructure of weldment on API $5 L \quad X-52$ steel pipe. Materials Characterization, 2007. 58(8-9): p. 721-729.

4. Haller, K., Nonlinear Acoustics Applied to NonDestructive Testing. 1st ed. 2007, Department of Mechanical Engineering, School of Engineering Blekinge Institute of Technology, Sweden: Blekinge Institute of Technology.

5. Lakhdar, M., et al., Damages Detection in a Composite Structure by Vibration Analysis. Energy Procedia, 2013. 36: p. 888-897.

6. Xu, H.L.H., Damage Detection for Structural Health Monitoring Using Ultrasonic Guided Waves, in School of Energy, Power and Mechanical Engineering. 2013, North China Electric Power University: Beijing, China.

7. Assunção E, C.L., Hagglund F, Troughton M, \& Spicer M, Advanced NDT techniques for plastic pipeline inspection. 2013, European Union under the Framework-7 TestPEP: EWF, Oeiras, Portugal.

8. Zenzinger, G., et al., Crack Detection Using EddyTherm. AIP Conference Proceedings, 2005. 760(1): p. 1646-1653.

9. Carvalho, A.A., et al., Reliability of non-destructive test techniques in the inspection of pipelines used in the oil industry. International Journal of Pressure Vessels and Piping, 2008. 85(11): p. 745-751.

10. Jenal, R., Fatigue crack detection using nonlinear acoustic - Analysis of vibro-acoustic modulations. , in Department of Mechanical Engineering. 2010, The University of Sheffield, United Kingdom.

11. C. Willberg, S.D., J.M. Vivar-Perez, Z.A.B. Ahmad, Simulation Methods for Guided Wave-Based Structural Health Monitoring: A Review. Applied Mechanics Reviews. January 2015. Vol. 67/0108031.

12. Ahmed, M.N., A Study of Guided Ultrasonic Wave Propagation Characteristics in Thin Aluminum Plate for Damage Detection, in Graduate Faculty. 2014, The University of Toledo: United States.

13. Shi Yan, J.Q., Nai-Zhi Zhao, Yang Cheng and Sheng-Wenjun Qi, Multiple Crack Detection of Pipes Using PZT-based Guided Waves, in School of Civil Engineering. 2014, Shenyang Jianzhu University, Shenyang Liaoning 110168, China. 